\title{
Review: short acting methylphenidate has short term efficacy in children and adolescents with attention deficit disorder
}

Sources of funding:

Therapeutics Initiative

University of British

Columbia; the British

Columbia Ministry for

Children and Families;

and the Cochrane

Collaboration

For correspondence:

Dr H M Schachter,

Children's Hospital of

Eastern Ontario

Research Institute,

Ottawa, Ontario,

Canada.

hschacht@uottawa.ca

Schachter HM, Pham B, King J, et al. How efficacious and safe is short-acting methylphenidate for the treatment of attention-deficit disorder in children and adolescents? A meta-analysis. CMAJ 2001 Nov 27;165:1475-88.

\section{QUESTION: What is the efficacy and safety of short acting methylphenidate for attention deficit disorder (ADD) in children and adolescents?}

\section{Data sources}

Studies in any language published between 1981 and 1999 were identified by searching Medline, EMBASE/ Excerpta Medica, PsycINFO, ERIC, CINAHL, HEALTHStar, Biological Abstracts, Current Contents,

Methylphenidate $v$ placebo in children and adolescents with attention deficit disorder*

\begin{tabular}{llll} 
Outcomes & Standardised mean difference (95\% Cl) \\
Hyperactivity index (teacher reported) & $0.78(0.64$ to 0.91$)$ & \\
\hline Hyperactivity index (parent reported) & $0.54(0.40$ to 0.67$)$ & \\
\hline Parent/self reported adverse events & Methylphenidate & Placebo & NNH (CI) \\
Decreased appetite & $45 \%$ & $14 \%$ & $4(3$ to 6$)$ \\
\hline Insomnia & $48 \%$ & $31 \%$ & $6(4$ to 13$)$ \\
\hline Headache & $18 \%$ & $13 \%$ & $17(10$ to 72$)$ \\
\hline Stomachache & $24 \%$ & $15 \%$ & $12(6$ to 84$)$ \\
\hline
\end{tabular}

*Abbreviations defined in glossary; $\mathrm{NNH}$ and $\mathrm{Cl}$ calculated from absolute risk difference data in article.

\section{COMMENTARY}

The meta-analysis by Schachter et al supports a robust body of literature showing the efficacy of methylphenidate for the acute treatment of ADHD disorders in children and adolescents. Given the continuing suspicion with which the diagnosis is viewed by some elements of the lay public, the results of this study are welcome.

The analysis identifies problems with the extant literature, such as generally small study sample sizes that include a preponderance of boys, the short duration of most studies, and outcome measures that may confound ADHD with externalising comorbid behaviours. Because of these limitations, the authors suggest that broad generalisations about the usefulness of methylphenidate should probably be avoided.

The results raise several issues. Firstly, the moderate effect size of 0.54 for parent reported hyperactivity appears less robust than in previous ADHD studies, which report overall effect sizes of $0.8-1.1 .{ }^{2}$ One explanation may be that 52 of the reviewed studies $(84 \%)$ used either once daily or twice daily dosing strategies. Immediate release (IR) methylphenidate may wear off before the child returns home after school, resulting in the larger effect sizes reported by teachers. The current standard of care is 3 daily doses to help the child in the after school hours. Secondly, the results show the need for studies of longer duration. To date, 2 controlled studies have reported stimulant benefits continuing for up to 14 months. ${ }^{23}$ Thirdly, although children treated with methylphenidate have higher rates of medication induced side effects, it is important to compare these risks with the risks of untreated ADHD. Clearly, untreated ADHD has far greater detrimental effects on the child's development than the side effects of stimulants. This must be appropriately framed for the child's parents when clinicians discuss potential stimulant side effects. Finally, the role of IR stimulants will diminish given that the standard treatment is changing to long acting, once daily stimulants.

Daniel F Connor, MD University of Massachusetts Medical School Worcester, Massachusetts, USA

1 Swanson JM, McBurnett K, Wigal T, et al. Stimulant medications and the treatment of children with ADHD. Advances in Clinical Child Psychology 1995;17:265-322.

2 A 14-month randomized clinical trial of treatment strategies for attention-deficit/ hyperactivity disorder. The MTA Cooperative Group. Multimodal Treatment Study of Children with ADHD. Arch Gen Psychiatry 1999;56:1073-86.

3 Gillberg C, Melander H, von Knorring AL, et al.Long-term stimulant treatment of children with attention-deficit hyperactivity disorder symptoms. A randomized, double-blind, placebo-controlled trial. Arch Gen Psychiatry 1997;54:857-64.
Dissertation Abstracts, Cochrane Library Trials Register, and Current Controlled Trials; reviewing bibliographies of included studies and pertinent reviews; and reviewing the files of content experts.

\section{Study selection}

Randomised placebo controlled trials were included if they assessed the effects of short acting methylphenidate in children $\leqslant 18$ years of age with a primary diagnosis of ADD (based on a systematic and reproducible method). Exclusion criteria were $n$ of 1 studies, participants with conditions that required specialised school and/or home environments (eg, mental retardation or autism), or participants receiving stimulants other than methylphenidate.

\section{Data extraction}

Data were extracted on characteristics of the trial, population, and intervention; behavioural efficacy (eg, primary outcomes of teacher and parent versions of the hyperactivity index [HI]); and adverse events. Quality of individual studies was assessed using the 3 item Jadad scale (randomisation, blinding, and follow up) and an index of concealment of treatment allocation.

\section{Main results}

62 trials $(\mathrm{n}=2897)$ met the selection criteria. Mean sample size was 47 . Median age of participants was 8.7 years (52 trials) and median percentage of boys was $88 \%$ (59 trials). 45 trials included participants with a homogeneous primary diagnosis of attention deficit hyperactivity disorder or ADD with hyperactivity (ADHD). Mean length of intervention was 3.3 weeks.

Children who received methylphenidate had reduced scores on both teacher and parent reported $\mathrm{HI}$ compared with those who received placebo (table). Similar results (albeit of variable and smaller magnitudes) were found for teacher reported clinical response, global indices, core features, and key externalising features; attention and emotional lability did not differ between groups. Variable and weaker results were found for parent reported clinical response, global indices, core features, and key externalising features; inattention, hyperactivity/impulsivity, and oppositional defiant behaviour did not differ between groups. Children who received methylphenidate had higher parent/self ratings of decreased appetite, insomnia, headache, and stomachache (table).

\section{Conclusions}

Short acting methylphenidate reduces some core and related clinical manifestations of attention deficit disorder in children and adolescents in the short term, but is associated with increased adverse events. No long term studies were found. 